> Les troubles du spectre de l'autisme (TSA) sont caractérisés par des déficits pragmatiques qui ne dépendent pas du niveau développemental, que celui-ci soit verbal ou non verbal. Cette synthèse expose, en trois volets, les directions récentes dans la recherche sur les déficits pragmatiques dans les TSA. Premièrement, nous discutons la façon dont un manque de sensibilité aux indices sociaux peut influencer l'acquisition lexicale. Deuxièmement, nous résumons des résultats récents qui suggèrent que la compréhension du langage non littéral pourrait être partiellement préservée dans les TSA. Troisièmement, nous décrivons les avancées dans l'étude des aptitudes conversationnelles dans l'autisme. <

Les troubles du spectre de l'autisme (TSA) consistent en une famille de déficits neurobiologiques de développement, dont on estime actuellement la prévalence à plus d'un pour cent de la population. Selon la $5^{e}$ édition du Diagnostic and statistical manual of mental disorders (DSM-V) [1], les TSA se caractérisent par une combinaison de difficultés sévères dans le domaine de l'interaction sociale avec des comportements rigides et stéréotypés. Alors que des éditions antérieures du DSM posaient différents sous-types d'autisme, notamment le syndrome d'Asperger et les troubles envahissants du développement non spécifiés (TEDNS), le DSM-V rassemble tous ces sous-types en une catégorie nosologique unifiée d'autisme, en opérant une distinction entre les individus qui présentent un retard significatif dans le développement du langage et ceux chez qui l'émergence du langage épouse une trajectoire typique. Le DSM-V a également introduit un changement au niveau de la définition des symptômes de l'autisme; il regroupe, sous l'étiquette de «déficit socio-pragmatique», d'une part, les problèmes dans la communication verbale et non verbale

Vignette (๔ Journée mondiale de sensibilisation à l'autisme).

\section{La pragmatique dans les troubles du spectre autistique}

\section{Développements récents}

Mikhail Kissine ${ }^{1}$, Elise Clin $^{1}$, Jessica de Villiers ${ }^{2}$

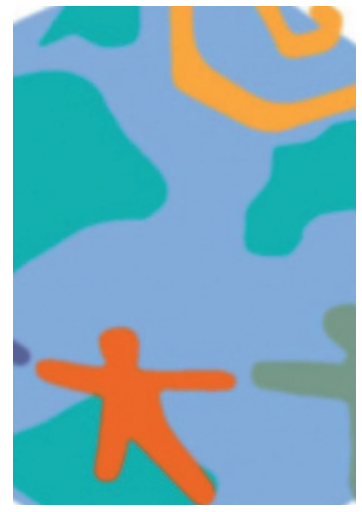

${ }^{1}$ ACTE/LaDisco, Université libre de Bruxelles, Bruxelles, Belgique ; ${ }^{2}$ University of British Columbia, Canada.

mkissine@ulb.ac.be

et, d'autre part, les aptitudes sociales déficientes - plutôt que de considérer, comme les définitions antérieures d'autisme, ces deux ensembles de symptômes comme distincts. L'unification de ces deux dimensions s'autorise du fait que, en dépit d'une grande variation dans les aptitudes proprement linguistiques, toutes les personnes avec TSA présentent des dysfonctionnements spécifiques au niveau de la pragmatique. Or, même si les acceptions du terme même de «pragmatique » peuvent varier au travers des diverses traditions de recherche, toutes font référence aux capacités cognitives requises pour une interaction sociale adéquate.

La recherche contemporaine connaît, bien sûr, des débats importants sur les corrélats cognitifs de l'autisme. Cependant, dans cette synthèse nous avons choisi de rester neutres par rapport à ces questions plus globales, afin de nous concentrer sur la réalité clinique des difficultés pragmatiques et, plus largement, communicationnelles dans les TSA. Une telle clarification n'est pas superflue, car, comme on le verra plus loin, la pragmatique dans l'autisme est, dans les faits, bien plus nuancée que le modèle d'un déficit plus ou moins global que la littérature scientifique prend souvent pour acquis.

\section{Pragmatique et acquisition lexicale dans les TSA}

Sous sa définition sans doute la plus répandue, la pragmatique se conçoit comme la capacité cognitive qui permet de traiter les stimulus communicationnels, verbaux ou non, en intégrant l'information contextuelle, y compris les intentions communicatives du locuteur. Cet objet de recherche présente une pertinence évidente pour l'étude de l'autisme; on le sait, depuis la fin des années quatre-vingt, une 
multitude d'études ont attesté des difficultés qu'éprouvent les enfants et les adultes avec TSA à adopter le point de vue d'une autre personne (par exemple [2]).

Il est important de souligner, à ce stade, que l'incapacité à accéder à la perspective de son interlocuteur n'affecte pas seulement des niveaux d'interprétation plus ou moins sophistiqués, tel le langage figuré (discuté plus loin), mais pourrait aussi expliquer en partie les fréquents retards dans le développement du langage qui caractérisent l'autisme. Tant la trajectoire de l'acquisition du langage que la taille du vocabulaire varient considérablement au travers du spectre de l'autisme. Toutefois, chez une très grande proportion d'enfants avec TSA les premiers mots n'apparaissent qu'avec un retard considérable; de façon encore plus dramatique, autour de $25 \%$ des individus avec autisme n'accèdent jamais au langage fonctionnel [3]. En parallèle, un programme de recherche fécond a révélé des difficultés très précoces chez des enfants avec autisme à établir et à maintenir un focus d'attention partagé avec des partenaires sociaux. En fait, ce manque de coordination attentionnelle constitue probablement un facteur prépondérant dans la trajectoire développementale atypique des enfants avec autisme (par exemple, [4]). Or, la capacité à suivre la direction du regard d'autrui, celle de contrôler l'attention conjointe et, plus généralement, de faire preuve de sensibilité aux indices sociaux sont autant de facteurs centraux dans l'acquisition du langage, que ce soit chez les enfants au développement typique ou dans les TSA [5-7]. Il se pourrait donc que les problèmes dans l'acquisition du langage dans les TSA soient partiellement causés par cette absence d'attention conjointe ou partagée. En d'autres termes, un manque très précoce de sensibilité à l'information «sociale » aurait un effet domino sur l'émergence du langage et la mise en place d'aptitudes communicationnelles.

Il est vrai que certains enfants sur le spectre autistique semblent tenir compte des gestes et du regard lors de l'apprentissage du langage, mais, de façon frappante, cette hétérogénéité dans la sensibilité aux indices sociaux semble refléter, du moins en partie, la variation dans la prédisposition même à acquérir le langage. Par exemple, alors que les bébés neuro-typiques exhibent une nette préférence pour la voix de leur mère (par rapport à un signal non linguistique analogue), de nombreux enfants sur le spectre ont tendance à n'exhiber aucune préférence allant dans ce sens, même si l'absence d'une telle orientation préférentielle n'est pas présente de façon uniforme dans l'autisme. Or, justement, ces enfants TSA qui ne font preuve d'aucun traitement distinctif des stimulus linguistiques manquent également de sensibilité aux caractéristiques spécifiques à la parole, comme, par exemple, la variation dans la durée des syllabes [8]. Dans le même ordre d'idées, il semblerait que les enfants TSA avec des symptômes sociaux sévères présentent aussi plus de réponses neurologiques atypiques en réaction au contraste entre des mots connus et inconnus [9].

Comme on vient de l'évoquer, certains jeunes enfants avec autisme réussissent à se baser sur la direction du regard de leur partenaire interactionnel pour coupler des mots nouveaux avec des objets inconnus. C'est d'autant plus le cas si ces enfants présentent un quotient intellectuel proche de la norme du développement typique, s’ils ne présentent pas de délai significatif dans le développement linguistique ou encore, si le regard du partenaire se voit combiné avec d'autres indices, tels que le pointage ou une interjection $[10,11]$. Toutefois, la variation individuelle dans la réceptivité face aux indices sociaux - certains enfants avec autisme y étant plus sensibles que d'autres - ne doit pas occulter la possibilité que ces processus soient, en réalité, de nature atypique. En effet, bien que des sous-groupes d'enfants TSA puissent diriger leur attention vers un objet en suivant le regard de leur partenaire, et apprendre ainsi le sens de mots nouveaux, leur traitement du regard d'autres personnes n'est peut-être pas de nature véritablement sociale. Des recherches récentes sur l'attention suggèrent que si certains enfants TSA utilisent, en effet, la direction des yeux dans des tâches qui requièrent de la flexibilité attentionnelle, ils sont aussi plus enclins que leurs pairs neuro-typiques à suivre un indice non social, tel qu'une flèche, au même degré qu'un regard $[12,13]$. En d'autres termes, il se pourrait que ce qui apparaît superficiellement comme de l'acquisition lexicale guidée par des indices sociaux relève, en réalité, d'un processus d'apprentissage associatif, qui ne privilégie aucunement l'information intrinsèquement sociale $[14,15]$.

En outre, le processus d'apprentissage lexical pourrait être plus «superficiel » chez les enfants avec autisme que dans le développement typique. En effet, certains enfants TSA arrivent à se baser sur le regard pour coupler des formes phonologiques (la forme sonore du mot) nouvelles avec des objets, mais ils ne parviennent pas toujours à intégrer l'information sémantique (le sens du mot) au sein de leur lexique mental, c'est-à-dire à associer le mot nouvellement acquis à une signification riche et flexible, interconnectée avec celles d'autres mots $[16,17]$. Cette hypothèse expliquerait pourquoi on observe souvent un vocabulaire en réception anormalement bas par rapport au vocabulaire expressif chez les enfants avec TSA, ainsi que le fait que l'utilisation de mots soit généralement figée et peu productive dans l'autisme [7, 18, 19].

\section{Au-delà du sens littéral}

Après avoir présenté la façon dont les déficits pragmatiques peuvent influencer le développement linguistique dans l'autisme, tournons-nous, à présent, vers les aspects pragmatiques du langage une fois que celuici a été (partiellement) acquis. L'autisme a souvent 
été associé à des difficultés dans la compréhension des métaphores, des requêtes indirectes ou de l'ironie. Une idée communément défendue - et présente dans les critères diagnostiques du DSM-V - est que des difficultés à attribuer des états mentaux aux autres personnes empêcheraient, de façon uniforme, les personnes avec TSA de tenir compte du contexte pour aller au-delà du sens strictement littéral des énoncés.

Cependant, il existe de solides raisons empiriques pour penser que les personnes TSA recrutent des informations contextuelles lors du traitement d'énoncés verbaux. Par exemple, le degré auquel les enfants avec ou sans TSA convoquent le contexte linguistique afin de résoudre des cas d'ambiguité lexicale dépend de leur niveau verbal et non du diagnostic [20]. De même, la compréhension des métaphores n'est pas spécifiquement déficitaire dans l'autisme, mais dépend des compétences sémantiques [21]. En fait, il est plausible qu'un lexique peu flexible et réduit - lui-même effet d'un déficit pragmatique précoce (voir plus haut) - puisse bloquer la modulation du sens lexical requise pour créer une interprétation métaphorique. Cependant, l'interprétation métaphorique peut se déclencher de façon spontanée chez des personnes TSA pourvues d'un niveau verbal élevé [22]. Dans le même ordre d'idées, une fois mises en place des conditions naturelles d'observation, des enfants avec TSA exhibent une compréhension véritablement contextuelle des requêtes indirectes $[23,24]$.

Bien entendu, la structure et la taille du lexique ou le caractère inadapté des paradigmes expérimentaux ne suffisent pas à expliquer toutes les difficultés avec le langage non littéral qui ont été attestées dans les TSA au fil des années. La compréhension de l'ironie, notamment, est largement déficitaire dans l'autisme [25]. Ce n'est que peu étonnant lorsqu'on sait que des capacités avancées d'attribution d'états mentaux sont requises pour comprendre l'ironie et que, en dépit de variations individuelles, ces capacités sont notoirement déficitaires dans l'autisme [26]. II est vrai que quelques études récentes ont suggéré que des adultes avec autisme parviennent à discriminer correctement les énoncés littéraux des énoncés sarcastiques $[27,28]$. Cependant, le niveau de chance dans ces études est de $50 \%$, avec des stimuli ironiques clairement marqués par une intonation distinctive et saillante; ces expériences montrent, certes, que les personnes avec TSA peuvent se baser sur des indices para-verbaux pour détecter l'ironie, mais sans doute pas que le sens ironique est dérivé à proprement parler.

\section{Conversation et interaction}

En complément aux aspects liés à la compréhension, les compétences pragmatiques sont également cruciales au niveau de la production et, plus spécifiquement, pour la gestion et le maintien des conversations, une source de troubles au quotidien pour des personnes avec autisme (et ce, indépendamment du niveau de langage structurel). Il existe un consensus selon lequel les personnes avec autisme éprouvent des difficultés à initier des sujets qui peuvent présenter un intérêt partagé pour tous les participants à la conversation, ainsi qu'à se désengager d'un sujet d'une façon qui soit consistante avec des indices linguistiques ou para-verbaux émis par les partenaires conversationnels [29]. Les contributions conversationnelles des personnes avec autisme peuvent aussi paraître «pédantes », c'est-à-dire figées et d'une technicité abusive; d'un point de vue structurel, de telles contributions frappent par une absence d'ellipses et, au sein des informations communiquées, par une absence de hiérarchisation en fonction de la pertinence potentielle vis-à-vis de l'interlocuteur [30].

Bien que longtemps attachée à la détection des déficiences et à la recherche des déficits cognitifs sousjacents, l'analyse des discours cliniques se focalise désormais sur la mise en évidence de leur fonctionnement propre, qu'il soit plus ou moins typique. Au niveau théorique, les discours ont été reconsidérés dans leur contexte intrinsèquement interactif : plutôt que d'envisager une prise de parole comme l'incarnation d'un fonctionnement cognitif individuel, chaque phrase devient le produit d'un processus collaboratif entre interlocuteurs, en elle-même capable d'exercer en retour une influence sur le contexte. Ce mouvement implique également une lutte contre l'aspect impressionniste de certaines descriptions: il ne s'agira plus seulement d'observer un comportement langagier et de l'étiqueter, mais de le quantifier, de l'intégrer à la dynamique générale du dialogue et d'envisager son rôle au sein de l'interaction [31, 32].

Dans un effort pour intégrer la complexité du contexte conversationnel, certains paradigmes de recherche visent à s'appuyer sur des structures écologiques. En effet, le caractère peu naturel d'un entretien sous forme de questionnaire, le manque de familiarité entre l'expérimentateur et la personne sur le spectre autistique, ainsi que l'étrangeté du contexte d'expérimentation sont autant de facteurs qui ont pu contribuer à la caractérisation des individus avec TSA comme peu enclins à entrer en relation avec autrui. L'analyse des conversations, et plus largement des interactions, qui impliquent des enfants et adultes avec autisme, cherche plus aujourd'hui à étudier les compétences effectives des individus du spectre autistique et à déterminer leurs stratégies compensatoires [33]. En outre, une analyse minutieuse, qui inclut des aspects interpersonnels, peut révéler que, pour une partie, ce qui est caractérisé comme une inadéquation conversationnelle dans l'intervention d'une personne avec autisme l'est en vertu d'une conception somme toute normative. La gestion du sujet de la conversation peut ainsi être cohérente et appropriée, étant donné les intérêts et 
le point de vue de la personne avec autisme, tout en étant difficile à saisir et à suivre pour son interlocuteur neuro-typique $[34,35]$.

\section{Conclusion}

Au terme de ce bref survol des dernières avancées dans l'étude de la pragmatique dans l'autisme, on peut épingler, d'une part, une réalité clinique plus nuancée que la doxa d'un déficit interactionnel global, mais aussi, d'autre part, une prise de conscience croissante de l'existence d'une relation plus forte entre les déficiences pragmatiques et le développement langagier en général.

Concernant le premier point, une position classique consistait jusqu'à peu à faire l'hypothèse que les individus avec TSA seraient cantonnés, dans leur compréhension du langage, à des interprétations entièrement décontextualisées et littérales, et ce en raison d'une difficulté ou d'une incapacité à attribuer des états mentaux à autrui. Cependant, nous l'avons vu, des études de plus en plus nombreuses suggèrent fortement que certaines capacités pragmatiques peuvent être préservées dans l'autisme - surtout, on peut le penser, lorsqu'il suffit de considérer le contexte d'un point de vue égocentrique [36]. Bien entendu, l'idée que la compréhension contextuelle, pragmatique des énoncés, n'est pas nécessairement déficiente dans son intégralité chez les enfants porteurs d'autisme - et, surtout, qu'il existe une distinction claire entre les aspects égocentriques et allocentriques doit encore transparaître de façon explicite dans les instruments de dépistage et de diagnostic (voir, par exemple, [37]). En outre, la communauté scientifique doit encore élucider les raisons de difficultés dans les très nombreux aspects de l'interaction qui impliquent, à un degré plus ou moins fort, d'adopter la perspective d'autrui. II pourrait, par exemple, s'agir d'un déficit exécutif plus général, situé particulièrement au niveau de la flexibilité cognitive $[36,38]$. D'autres auteurs invoquent un manque intrinsèque de motivation sociale [39], même si certaines études révèlent une conscience du partenaire conversationnel et une véritable volonté de transmettre un fil narratif [35, 40, 41]. Quant à l'impact de la pragmatique sur les autres domaines linguistiques, il est de plus en plus évident que l'acquisition du langage repose sur des aptitudes interactionnelles qui impliquent la prise en compte d'autrui [5]. Or, on l'a vu, chez des enfants avec autisme un manque d'engagement dans des dynamiques d'attention partagée pourrait compromettre l'accès à l'apprentissage des premiers mots. À nouveau, les causes de cette sensibilité aux indices sociaux restent à élucider. Certains y voient un manque de préférence inné pour les visages humains, qui entraînerait, dans un effet domino, un manque d'expertise dans la détection des expressions faciales [42]. D'autres études, pourtant, ne trouvent aucune différence entre bébés avec TSA et ceux au développement typique au niveau de la quantité d'attention visuelle accordée aux visages et aux yeux dans les tout premiers mois de vie ; cependant, autour de 12 mois, le temps passé à fixer les visages décroît sensiblement chez les bébés avec autisme [43, 44].

On l'aura compris: même si des déficits socio-pragmatiques constituent indubitablement une caractéristique centrale de l'autisme, leur nature, leurs conséquences sur le développement et leurs corrélats neurocognitifs constituent un domaine de recherche crucial, qui exige un effort interdisciplinaire continu. $\diamond$

\section{SUMMARY}

Pragmatics in autism spectrum disorder: recent developments

Autism spectrum disorder (ASD) is characterized by primary pragmatic difficulties, out of step with verbal and non-verbal developmental level. This selective survey paper addresses three recent domains of research on pragmatic functions in autism. First, we provide an upto-date discussion of how lack of sensitivity to social cues impacts early acquisition of words. Second, we review recent findings on the comprehension of nonliteral language, pointing to a more refined clinical reality. Third, we describe recent developments in the study of conversation skills in autism. $\diamond$

\section{LIENS D'INTÉRÊT}

Les auteurs déclarent n'avoir aucun lien d'intérêt concernant les données publiées dans cet article.

\section{RÉFÉRENCES}

1. American Psychiatric Association. Diagnostic and statistical manual of mental disorders (DSM-5). Washington DC : APA, 2013

2. Yirmiya $N$, Erel 0 , Shaked $M$, Solomonica-Levi D. Meta-analyses comparing Theory of Mind abilities of individuals with autism, individuals with mental retardation, and normally developing individuals. Psychol Bull 1998; 124 : 283-307.

3. Tager-Flusberg H, Paul R, Lord C. Language and communication in autism. In : Volkmar FR, Paul R, Klin A, Cohen D, eds. Handbook of autism and pervasive developmental disorders, $3^{\text {rd }}$ ed. Hoboken, NJ : Wiley, 2005 : 335-405.

4. Mundy P, Gwaltney M, Henderson H. Self-referenced processing, neurodevelopment and joint attention in autism. Autism Int J Res Pract $2010 ; 14: 408-29$.

5. Tomasello M. Origins of human communication. The Jean Nicod lectures 2008. Cambridge, MA : MIT Press, 2008 : xiii : 394 p.

6. Mundy P, Sigman M, Kasari C. The Theory of Mind and joint-attention deficits in autism. In : Baron-Cohen S, Tager-Flusberg H, Cohen DJ, eds. Understanding other minds: perspectives from autism. Oxford : Oxford University Press, 1993 : 181-203.

7. Luyster RJ, Kadlec MB, Carter A, Tager-Flusberg H. Language assessment and development in toddlers with Autism Spectrum Disorders. J Autism Dev Disord 2008 ; 38 : 1426-38.

8. Kuhl PK, Coffey-Corina S, Padden D, Dawson G. Links between social and linguistic processing of speech in preschool children with autism: behavioral and electrophysiological measures. Dev Sci 2005 ; 8 : Fl-12.

9. Kuhl PK, Coffey-Corina S, Padden D, et al. Brain responses to words in 2 -year-olds with autism predict developmental outcomes at age 6. PLoS One $2013 ; 8$ : e64967.

10. Luyster R, Lord C. Word learning in children with autism spectrum disorders. Dev Psychol 2009 ; 45 : 1774-86.

11. Preissler MA, Carey $S$. The role of inferences about referential intent in word learning: evidence from autism. Cognition $2005 ; 97$ : B13-23.

12. Ristic J, Mottron L, Friesen CK, et al. Eyes are special but not for everyone: the case of autism. Cogn Brain Res $2005 ; 24: 715-8$.

13. Senju A, Southgate V, Miura Y, et al. Absence of spontaneous action anticipation by false belief attribution in children with autism spectrum disorders. Dev Psychopathol $2010 ; 22$ : 353-60. 


\section{RÉFÉRENCES}

14. McGregor KK, Rost G, Arenas R, et al. Children with ASD can use gaze in support of word recognition and learning. J Child Psychol Psychiatry $2013 ; 54: 745-53$.

15. Parish-Morris J, Hennon EA, Hirsh-Pasek K, et al. Children with autism illuminate the role of social intention in word learning. Child Dev $2007 ; 78$ : 1265-87.

16. Henderson L, Powell A, Gareth Gaskell M, Norbury C. Learning and consolidation of new spoken words in autism spectrum disorder. Dev Sci $2014 ; 17: 858-71$.

17. Norbury CF, Griffiths H, Nation K. Sound before meaning: word learning in autistic disorders. Neuropsychologia $2010 ; 48: 4012-9$.

18. Loucas T, Charman T, Pickles A, et al. Autistic symptomatology and language ability in autism spectrum disorder and specific language impairment. J Child Psychol Psychiatry $2008 ; 49$ : 1184 92.

19. Perkins MR, Dobbinson S, Boucher J, et al. Lexical knowledge and lexical use in autism. J Autism Dev Disord $2006 ; 36: 795-805$

20. Brock J, Norbury CF, Einav $S$, Nation K. Do individuals with autism process words in context? Evidence from language-mediated eye-mouvements. Cognition 2008 ; 108 : 896-904.

21. Norbury CF. The relationship between Theory of Mind and metaphor: evidence from children with language impairement and autistic spectrum disorder. Br J Dev Psychol $2005 ; 23$ : 383-99.

22. Hermann I, Haser V, Van Elst LT, et al. Automatic metaphor processing in adults with Asperger syndrome: a metaphor interference effect task. Eur Arch Psychiatry Clin Neurosci $2013 ; 263$ (suppl 2) : S177-87.

23. Kissine M, Cano-Chervel J, Carlier S, et al. Children with autism understand indirect speech acts: evidence from a semi-structured act-out task. PLoS One 2015 ; 10 : e0142191.

24. Kissine $M$, De Brabanter $P$, Leybaert J. The interpretation of requests in children with autism: the effect of the sentence-type. Autism $2012 ; 16: 523-32$.

25. Martin I, McDonald S. An exploration of causes of non-literal language problems in individuals with Asperger syndrome. J Autism Dev Disord 2004 ; 34 : 311-28.

26. Happé FGE. The role of age and verbal ability in the Theory of Mind task performance of subjects with autism. Child Dev $1995 ; 66: 843-55$.

27. Chevallier C, Noveck I, Happé FGE, Wilson D. What's in a voice? Prosody as a test case for the theory of mind account of autism. Neuropsychologia $2011 ; 49: 507-17$.

28. Colich NL, Wang AT, Rudie JD, et al. Atypical neural processing of ironic and sincere remarks in children and adolescent with autism spectrum disorders. Metaphor Symb $2012 ; 27: 70-92$.

29. De Villiers J, Fine J, Ginsberg G, et al. A scale for rating conversational impairment in autism spectrum disorder. J Autism Dev Disord 2007 ; 37 : 1375-80.

30. Baltaxe CAM, D'Angiola N. Cohesion in the discourse interaction of autistic, specifically languageimpaired, and normal children. J Autism Dev Disord 1992 ; 22 : 1-21.
31. Asp $\varepsilon$, de Villiers J. When language breaks down. Analysing discourse in clinical contexts. Cambridge : Cambridge University Press, 2010.

32. Sterponi L, de Kirby K, Shankey J. Rethinking language in autism. Autism $2014 ; 19: 517-26$.

33. Plumet $M H$, Veneziano $\varepsilon$. Typical and atypical pragmatic functioning of ASD children and their partners: a study of oppositional episodes in everyday interactions. J Autism Dev Disord $2014 ; 45$ : 53-67.

34. Muskett T, Perkins MR, Clegg J, Body R. Inflexibility as an interactional phenomenon: using conversation analysis to re-examine a symptom of autism. Clin Linguist Phon 2010 ; $24: 1-16$.

35. De Villiers J. I saw the yellowish going south: narrative discourse in autism spectrum disorder. Belg J Linguist $2011 ; 25: 3-29$.

36. Kissine M. Pragmatics, cognitive flexibility and autism spectrum disorders. Mind Lang $2012 ; 28: 1-28$.

37. Paul R. Assessing communication inautism spectrum disorders. In : Volkma FR, Paul R, Klin A, Cohen D, eds. Handbook of autism and pervasive developmental disorders, $3^{\text {rd }}$ ed. Hoboken, NJ : Wiley, $2005: 799-816$.

38. Hill $\varepsilon$. Executive dysfunction in autism. Trends Cogn Sci $2004 ; 8: 26-32$.

39. Chevallier C, Kohls G, Troiani V, et al. The social motivation theory of autism. Trends Cogn Sci $2012 ; 16: 231-9$.

40. Volden J, Sorenson A. Bossy and nice requests: Varying language register in speakers with autism spectrum disorder (ASD). J Commun Disord $2009 ; 42$ : 58-73.

41. Hobson R, Hobson J, García-Pérez R, Du Bois J. Dialogic linkage and resonance in autism. J Autism Dev Disord $2012 ; 42: 2718-28$.

42. Schultz RT. Developmental deficits in social perception in autism: the role of the amygdala and fusiform face area. Int J Dev Neurosci $2005 ; 23: 125-41$.

43. Jones $\mathrm{W}, \mathrm{Klin} \mathrm{A}$. Attention to eyes is present but in decline in $2-6$-month-old infants later diagnosed with autism. Nature 2013 ; 504 : 427-31.

44. Ozonoff S, losif AM, Baguio F, et al. A prospective study of the emergence of early behavioral signs of autism. J Am Acad Child Adolesc Psychiatry 2010 ; 49 : 256-66.

\section{TIRÉS À PART}

M. Kissine

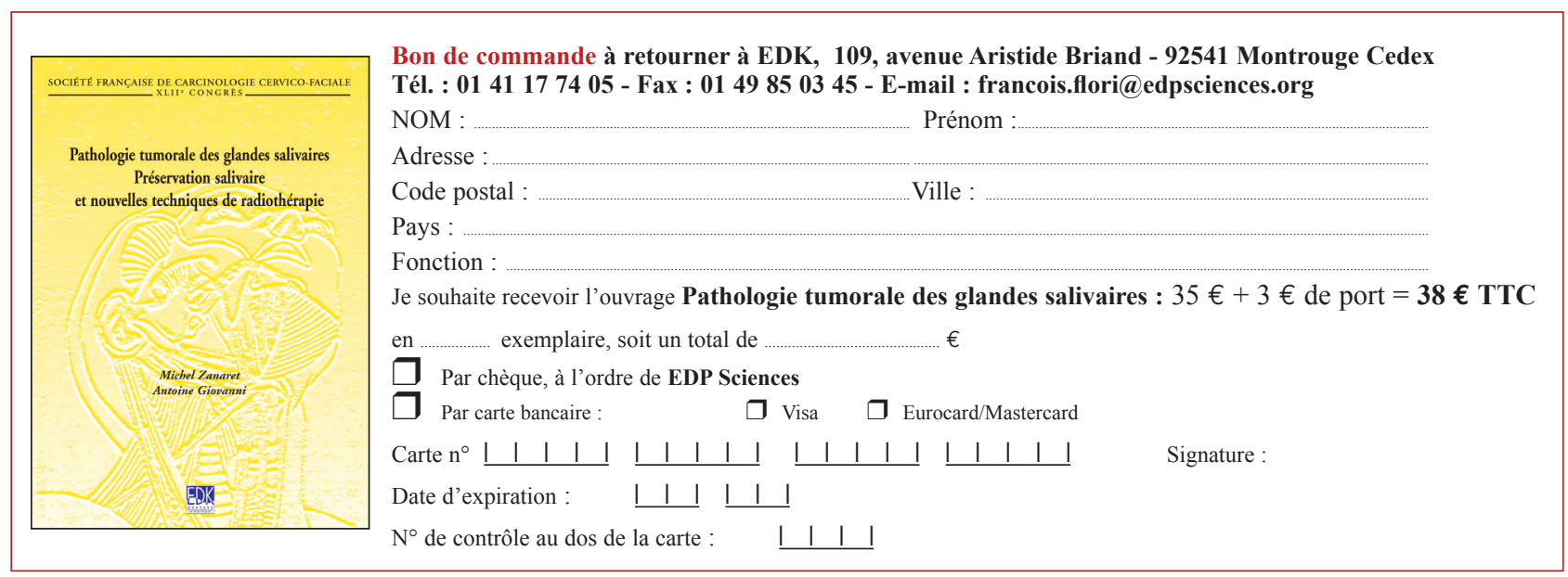

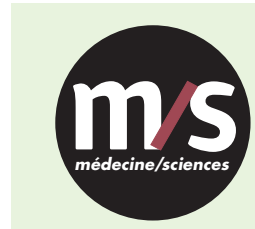

Tarifs d'abonnement $\mathrm{m} / \mathrm{s}-2016$

Abonnez-vous

à médecine/sciences
$>$ Grâce à $m / s$, vivez en direct les progrès des sciences biologiques et médicales

\section{Bulletin d'abonnement} page 910 dans ce numéro de $\mathrm{m} / \mathrm{s}$

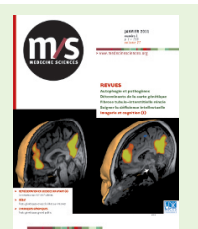

